\title{
Quantum versus semiclassical analysis of the conductivity of two-dimensional electrons in a magnetic field
}

\author{
M. Hayne \\ Department of Physics, University of Exeter, Stocker Road, Exeter EX4 4QL, United Kingdom \\ and Laboratorium voor Vaste-Stoffysica en Magnetisme, Katholieke Universiteit Leuven, Celestijnenlaan 200D, B-3001 Leuven, Belgium
}

A. Usher

Department of Physics, University of Exeter, Stocker Road, Exeter EX4 4QL, United Kingdom

J. J. Harris

Department of Electronic and Electrical Engineering, University College, London WC1E 7JE, United Kingdom

C. T. Foxon

Department of Physics, University of Nottingham, University Park, Nottingham, NG7 2RD, United Kingdom

(Received 1 April 1997)

\begin{abstract}
We present experimental evidence for the levitation of extended states of a two-dimensional electron system in a magnetic field, and establish the presence of alternating regions of localized and extended states in the density of states to Landau-level filling factors $(\nu)$ as high as 80 . Monitoring the Hall voltage of a modulationdoped $\mathrm{GaAs} / \mathrm{Al}_{x} \mathrm{Ga}_{1-x} \mathrm{As}$ heterojunction at fixed magnetic field during continuous illumination from lightemitting diodes reveals a steplike structure with plateaus at even integer $\nu$ between 22 and 80 at a temperature of $0.3 \mathrm{~K}$. We derive a general expression for the conductivity due to rectangular bands of extended states, and show that the observed temperature dependence of the Shubnikov-de Haas oscillations is consistent with this picture. An analysis of the oscillations using this expression reveals the predicted levitation of the extended states. [S0163-1829(97)05140-0]
\end{abstract}

\section{INTRODUCTION}

For several years the temperature, $T$, and magnetic field, $B$, dependences of low $B$ Shubnikov-de Haas (SdeH) oscillations have been used to determine properties such as the effective mass, $m^{*}$, and the single-particle scattering time, $\tau_{q}$, of two-dimensional (2D) electrons in $\mathrm{GaAs} / \mathrm{Al}_{x} \mathrm{Ga}_{1-x} \mathrm{As}$ heterojunctions. This is usually achieved by comparing the peak-to-peak amplitude of $\mathrm{SdeH}$ oscillations, $2 \Delta \rho_{x x}$, with the expression ${ }^{1}$

$$
\Delta \rho_{x x}=4 \rho_{0} D(\chi) \exp \left(\frac{-\pi}{\omega_{c} \tau_{q}}\right) \cos \left(\frac{2 \pi E_{f}}{\hbar \omega_{c}}-\pi\right),
$$

where

$$
D(\chi)=\frac{\chi}{\sinh \chi}
$$

(with $\chi=2 \pi^{2} k T / \hbar \omega_{c}$ ) describes the thermal damping of the oscillations, and $\rho_{0}$ is the zero-field resistivity. $E f$ is the Fermi energy, $\omega_{c}$ is the cyclotron frequency, $\hbar$ is the Planck constant divided by $2 \pi$, and $k$ is the Boltzmann constant. Recently this analysis has been extended to the extreme quantum limit where oscillations around Landau-level filling factor, $\nu$, of $1 / 2$ are interpreted as being due to composite fermions in an effective field, $B^{*}=B-B(\nu=1 / 2)$, where $B(\nu=1 / 2)$ is the magnetic field corresponding to $\nu=1 / 2 .^{2-4}$ Such analyses depend on the semiclassical picture of the density of states DOS in which the zero- $B$ 2D DOS gradu- ally evolves into Gaussian or Lorentzian-shaped Landau levels with widths determined by $\tau_{q}$. In this approach there are no localized states; thus while the system is metallic at $B$ $=0$, consistent with what is observed experimentally, for high-mobility samples, the observation of quantum Hall plateaus cannot be explained.

The quantum picture of the 2D DOS is in rather stark contrast to this, with a system that is localized at $B=0$, and at high-field consists of one, or a band of, extended states at the center of each Landau level with localized states in the tails. Such a configuration is widely accepted as the basis for explaining the quantum Hall effect. ${ }^{5}$ The transition between the $B=02$ D DOS in which all the occupied states are localized, and the high-field 2D DOS is achieved by a process in which the extended states, which are above the Fermi energy at $B=0$ "ffloat down" towards the center of the Landau levels as they become resolved. ${ }^{6,7}$ Experimental evidence for this process has been reported for low-mobility 2D electron systems ${ }^{8}$ (2DES) by identifying the peaks in the longitudinal conductivity with the position of the extended states. A recent theory by Haldane and Yang, ${ }^{7}$ which attributes the microscopic origin of the levitation to Landaulevel mixing suggests a different interpretation. They find that not only do the extended states move upwards in energy from $\left(n+\frac{1}{2}\right) \hbar \omega_{c}$ (where $n$ is the Landau-level index) by an amount proportional to $\left(n+\frac{1}{2}\right) / B^{3}$, but that there is also a shift of the mean Landau-level energy downwards of an order of $1 / B^{2}$, such that the extended states are brought towards the high-energy tail of the Landau level. Since the 


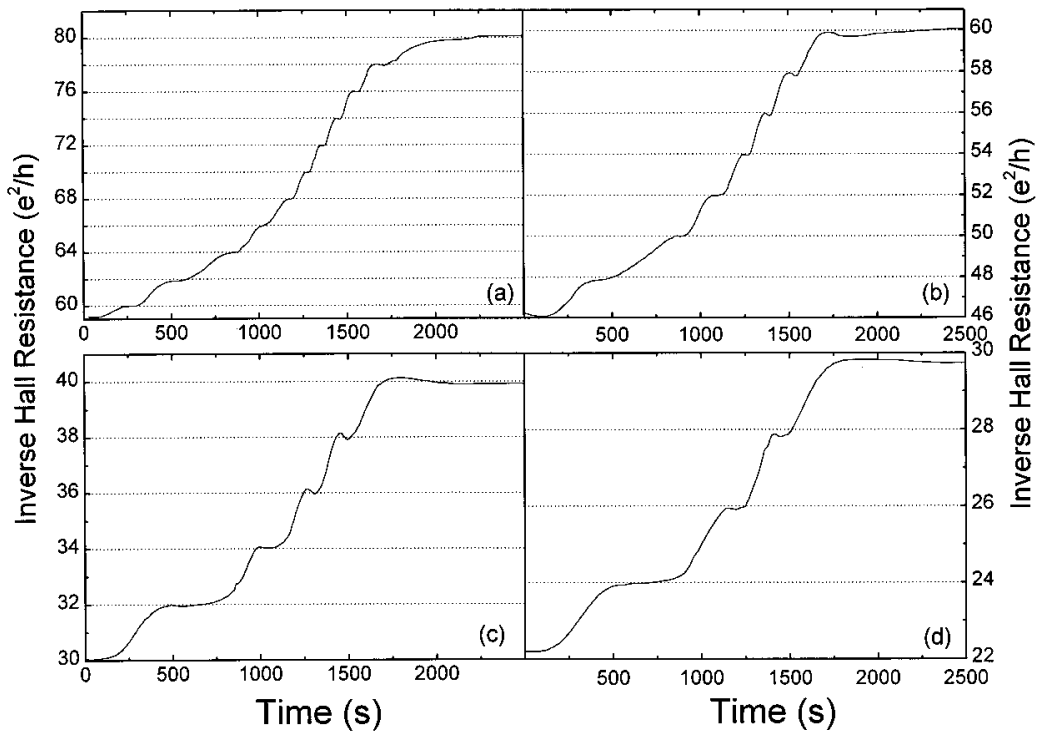

FIG. 1. Inverse Hall resistance in units of $e^{2} / h$ versus illumination time with an IRLED at $0.3 \mathrm{~K}$, and magnetic fields of (a) $0.15 \mathrm{~T}$, (b) 0.2 $\mathrm{T}$, (c) $0.3 \mathrm{~T}$, and (d) $0.4 \mathrm{~T}$. latter of these dominates at large $B$ and small $n$, they suggest that it, and not the levitation, may be the origin of the results reported in Ref. 8 .

In general, the semiclassical picture has been used, with apparent success, in the interpretation of $\mathrm{SdeH}$ oscillations at low $B$, whereas the quantum picture is necessary to describe the onset of the quantum Hall effect. However, there seems to be no clear criterion determining the regimes in which the use of each of these descriptions is justified. This contradiction is highlighted by the use of a semiclassical description of the 2D DOS of composite fermions, in a regime in which the electrons are highly nonclassical.

\section{EXPERIMENTAL RESULTS}

For a range of static magnetic fields between 0.15 and 0.4 $\mathrm{T}$, we have measured the variation in the Hall voltage of a 400- $\AA$ spacer $\mathrm{GaAs} / \mathrm{Al}_{x} \mathrm{Ga}_{1-x}$ As heterojunction [mobility $\left.(1.5-2.3) \times 10^{6} \mathrm{~cm}^{2} / \mathrm{V} \mathrm{s}\right]$ while changing the $2 \mathrm{D}$ density with illumination from a red $(R)$ or an infrared (IR) lightemitting diode (LED). The LED's were placed such that the Hall-bar sample was uniformly illuminated.

We find that, even at the modestly low temperature of 0.3 $\mathrm{K}$, we observe a steplike increase in the 2D density, resolving plateaus with $\nu$ as high as 80 . Figures $1(\mathrm{a})-1(\mathrm{~d})$ show the inverse Hall resistance in units of $e^{2} / h$ as a function of illumination time with an IRLED. As with all the data presented here, the sample was continuously illuminated by passing a current of $1 \mu \mathrm{A}$ through the LED. It can be seen from this figure that the Hall resistance has a steplike dependence on the illumination time, with plateaus that correspond to even integer $\nu$. (At such low $B$ there are no odd integer plateaus since the spin splitting of the Landau levels is not resolved.)

At long illumination times the plateaus develop small oscillatory features, which we attribute to mixing between the longitudinal and transverse components of the resistivity in the sample, $\rho_{x x}$ and $\rho_{x y}$, respectively. As might be expected for mixing, these oscillations become more clearly resolved at higher $\nu$ where $\rho_{x x}$ becomes comparable in size to $\rho_{x y}$. Coupled with this is the increase in the sample mobility which accompanies the illumination process, as a result of neutralization of $\mathrm{Si}$ donors in the $\mathrm{Al}_{x} \mathrm{Ga}_{1-x} \mathrm{As} .{ }^{9}$ This increases the size of the SdeH oscillations without affecting the size of the Hall voltage for a given 2D density and magnetic field. A third contributory factor to the size of the oscillatory features is sample inhomogeneity, which is evident from conventional SdeH oscillations in the dark, but is removed by illumination. This also increases the relative size of $\rho_{x x}$. It should be noted, however, that despite the fact that these oscillations are clearly resolved, they represent less than $1 \%$ of the measured Hall voltage in the worst case.

The same plateau structure is also observed when the sample is illuminated with a RLED, as can be seen in Fig. 2, where we present data at $0.3 \mathrm{~T}$ for temperatures of $0.3,0.68$, and $1.3 \mathrm{~K}$. For RLED illumination we initially observe a small increase in the 2D density, followed by a large persistent drop, before it finally increases significantly. As might be expected, we can see plateaus twice for the same $\nu$, once while the density is dropping, and once while it increases again. At $0.3 \mathrm{~K}$ there are well-defined plateaus at shorter illumination times, and stronger mixing at longer illumination times. At $0.68 \mathrm{~K}$ the plateaus at short illumination times have almost disappeared, whereas at long illumination times

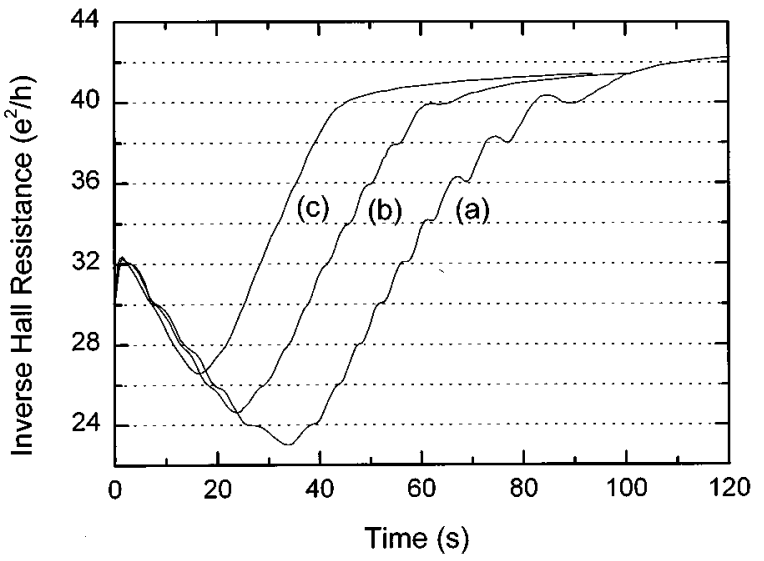

FIG. 2. Inverse Hall resistance in units of $e^{2} / h$ versus illumination time with a RLED at $0.3 \mathrm{~T}$, and temperatures of (a) $0.3 \mathrm{~K}$, (b) $0.68 \mathrm{~K}$, (c) $1.3 \mathrm{~K}$. 
the mixing in the higher $\nu$ plateaus observed at $0.3 \mathrm{~K}$ has gone, a result of the thermal damping of the SdeH oscillations. At $1.3 \mathrm{~K}$ the structure has all but disappeared.

In discussing the above results it is necessary to consider the means by which electrons released from the $D X$ centers $^{10}$ in the $\mathrm{Al}_{x} \mathrm{Ga}_{1-x} \mathrm{As}$ by the phenomenon of persistent photoconductivity get to the 2DES. For samples with a sufficiently narrow undoped spacer layer between the doped part of the barrier and the channel, the electrons tunnel through the triangular barrier in the spacer layer. In such samples, the rate of increase of 2D density with illumination is seen to drop exponentially, simply as a result of the decreasing probability of finding a $D X$ center to ionize. ${ }^{9}$ However, for samples where the spacer layer is wide, such as the one studied here, the tunneling process is very slow, and electrons are transferred from the $\mathrm{Al}_{x} \mathrm{Ga}_{1-x} \mathrm{As}$ to the 2DES via the contacts. Despite this, it is important to realize that the rate at which electrons are released from $D X$ centers must still decrease exponentially with time. In the case of the data of Fig. 1, it can be seen that the rate of increase of the 2DES density is initially very slow, and gets faster with more illumination. From this contradictory behavior, we can see that there is some "bottleneck" which limits the rate at which the electrons transfer from the $\mathrm{Al}_{x} \mathrm{Ga}_{1-x}$ As to the 2DES.

One possible explanation of the plateaus is that this bottleneck is $\nu$ dependent, such that the rate of increase of the 2DES density fluctuates. In support of this we find that the structure in the Hall resistance of conventional experiments in which the field is swept at constant density is not as well resolved as that of Figs. 1 and 2, even when the rate of change of Hall voltage is slower in the conventional experiment. The exact mechanism by which the Hall plateaus might be enhanced by this type of experiment is not understood at present, but some residual reduction in the ability of localized states to transfer charge seems possible. Another explanation is that the plateaus are a direct observation of the quantum Hall effect, and occur when $E_{f}$ passes through localized states in the tail of each Landau level. In fact, unless there is no change in density in the plateaus, the first explanation also requires the presence of the quantum Hall effect. Unfortunately, we are not able to verify whether the change in density is smooth or steplike by measuring the Hall coefficient at lower $B$. This is for two reasons. First, due to the presence of the bottleneck there is a reservoir of charge in the $\mathrm{Al}_{x} \mathrm{Ga}_{1-x} \mathrm{As}$ which will continue to transfer to the 2DES. Second, in the event that the bottleneck is $\nu$ dependent sweeping the magnetic field would change the experimental conditions. Besides, whichever of these effects is dominant is not crucial for our investigation, since they both invoke the quantum picture, and are not consistent with the semiclassical one. It would be quite implausible to suggest that that the plateaus can simply be explained by an oscillating DOS. In this case plateaus in the Hall resistance would mean that there is no change in 2D density, and therefore that there is a gap in the DOS. At such low field this is very hard to justify, especially bearing in mind that the amplitude of the oscillations in $\rho_{x x}$ are still small. For example, after illumination and at $0.3 \mathrm{~K}$ (when the oscillations are at their largest) the ratio $\Delta \rho_{x x} / \rho_{0}$ is $0.3,0.11$, and 0.049 at $0.3,0.2$, and $0.15 \mathrm{~T}$, respectively.

We therefore conclude that, even at these high $\nu$, the

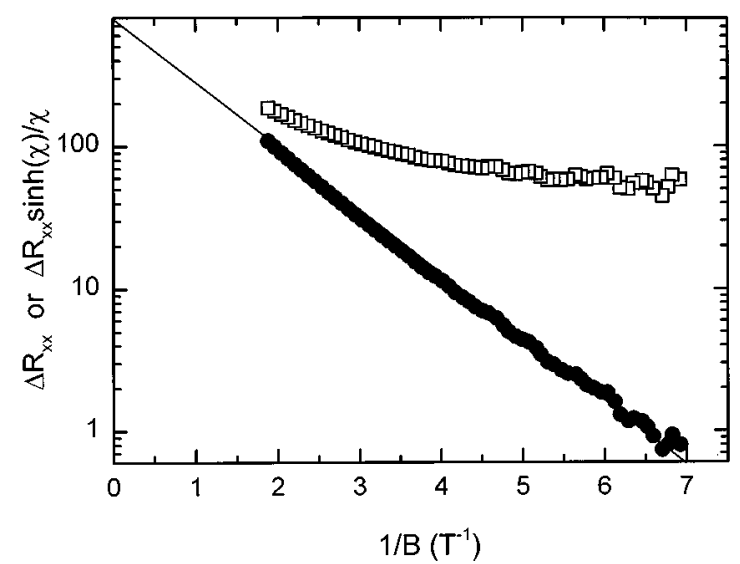

FIG. 3. "Dingle plot" for data from our earlier report (Ref. 11). The closed circles show the data with the invalid approximation that $\chi<1$, and the open squares have the standard temperature correction included. It can be seen that the standard analysis produces a poor Dingle plot, whereas the data uncorrected for temperature produces a linear dependence with the correct intercept.

quantum picture of the 2D DOS, in which there are extended states near the center of the Landau levels, with localized states in the tails should be used. Such a situation is inconsistent with the semiclassical description of the SdeH oscillations given by Eq. (1). Indeed, it has already been noted that the $T$ dependence of Eq. (1) does not seem to work in the case of samples such as the one studied here, ${ }^{11,12}$ though the reason for this has not been clear. To demonstrate this we show in Fig. 3 some of the data from another $400-\AA$ spacer $\mathrm{GaAs} / \mathrm{Al}_{x} \mathrm{Ga}_{1-x} \mathrm{As}$ heterojunction studied previously, ${ }^{11}$ where we have plotted $\Delta \rho_{x x} \sinh (\chi) / \chi$ versus $1 / B$ on a semi$\log$ plot for $T=1 \mathrm{~K}$ (a traditional "Dingle plot'). The effect of plotting this data "correctly," i.e., by dividing the oscillation amplitude by the thermal damping term $D(\chi)$, is shown by the open squares in the figure. This increases the relative size of the oscillations at low $B$, such that the curve is not linear, and if the lower-field portion is taken, has an incorrect intercept. The effect of incorporating the thermal damping term is thus to reduce the slope of the low-field portion, resulting not only in curved Dingle plots, but also in what we earlier acknowledged to be the highly improbable result that $\tau_{q}$ increases with temperature. ${ }^{11}$ For comparison, we show the effect of neglecting the thermal damping term $D(\chi)$ in Eq. (1) by assuming that it is 1 , an assumption that is only supposed to be valid when $\chi \ll 1$ (solid dots). For GaAs it happens that $\chi \approx T / B$, so this assumption should be a very poor one, and the oscillation amplitude should be increasingly damped at lower $B$. However, this is not the case. In fact, the plot is linear over a wide range of $1 / B$, and even has a correct intercept. This implies that the thermal damping of the oscillations either has an exponential field dependence, or none at all. A similar failure of the temperature dependence of Eq. (1) has been observed in other samples, ${ }^{12}$ and so casts serious doubts on the use of the semiclassical model.

\section{ANALYSIS}

In the previous section we reported the observation of plateaus in the Hall resistance at very high filling factors, and 
showed that this is indicative of alternating regions of extended and localized states in the 2D DOS, and inconsistent with the semiclassical picture used in the derivation of Eq. (1). We now propose a revised description of the SdeH oscillations, and demonstrate that it is generally consistent with experimental observations. We start by considering each Landau level to consist of a rectangular region of extended states at the center and localized states in the tails. The use of a band rather than a single discreet extended state can be justified on several accounts. First, although it is known that the width of the conductivity peaks diminishes with decreasing temperature, this effect saturates due to finite sample size. ${ }^{13}$ It has also been suggested that for high-mobility 2DES the presence of Coulomb interactions will result in a finite width of extended states at $0 \mathrm{~K} .{ }^{14}$ Finally, for the case of SdeH oscillations in which the spin splitting is unresolved, a similar delocalization process is expected as a result of the spin-orbit interaction. ${ }^{15}$ Using the expression for the temperature dependence given by Smrčka and Streda, ${ }^{16}$ the contribution to the longitudinal conductivity from the extendedstate region of the $n$th Landau level with width $2 \Delta E$ and centered at an energy $E_{n}=(n+1 / 2) \hbar \omega_{c}$ is given by

$$
\begin{aligned}
\sigma_{x x}^{n}= & -\int_{E_{n}-\Delta E}^{E_{n}+\Delta E} \sigma_{0} \frac{\partial f(E)}{\partial E} d E \\
= & \frac{\sigma_{0} \sinh \left(\frac{\Delta E}{k T}\right)}{\cosh \left(\frac{E_{n}-E_{f}}{k T}\right)+\cosh \left(\frac{\Delta E}{k T}\right)},
\end{aligned}
$$

where $\sigma_{0}$ is the Landau-level peak conductivity, which is assumed to be constant for a given $B$, and $f(E)$ is the FermiDirac distribution function. The total conductivity due to the extended states is then given by the sum over all Landau levels. There will also be a contribution to the conductivity from the localized states in the tails, which will be discussed below. At present it is useful to make some observations about Eq. (4). First, it is obtained by fundamental considerations of conductivity. Second, it can be applied independently to both maxima and minima. Strictly speaking, it is not valid in between because we have assumed $E_{f}$ to be $T$ independent, which is true at a maximum or minimum, where the DOS is symmetric about $E_{f}$. However, if the modulation of the total DOS is very weak, as is the case at low $B$, this would be a good approximation. Indeed, the same approximation is used in the semiclassical approach. ${ }^{12}$ Third, it is valid in high fields where the SdeH oscillations bottom out and Eq. (1) predicts oscillations which dip below zero $\left(\Delta \rho_{x x}>\rho_{0}\right)$. We can apply Eq. (4) to this case by taking the limit of high field and low temperature $\left(E_{n}-E_{f} \gg k T\right.$, $\Delta E \gg k T)$ in which we need only consider the contribution to the conductivity from the Landau levels nearest to $E_{f}$. In a minimum the conductivity due to the two adjacent levels ( $n=m$ and $n=m-1$ ), assumed to be equally far from $E_{f}$, is

$$
\sigma_{x x}^{\min }=\frac{2 \sigma_{0}}{\exp \left(\frac{E_{n}-E_{f}-\Delta E}{k T}\right)+1},
$$

which, in the same limit, approximates to the familiar activation formula over a gap of $E_{m}-E_{f}-\Delta E$, provided that $\Delta E \ll E_{m}-E_{f}$. It is worth noting that the usual assumption that activated conductivity is simply proportional to the occupancy of the excited level (see, for example, Ref. 17) fails to explain the fact that the conductivity saturates above temperatures with $k T \sim\left(E_{m}-E_{f}-\Delta E\right) / 10,{ }^{17}$ much lower than would be expected from the simple activation formula. In contrast, our treatment does predict this effect: when $E_{m}-E_{f} \gg k T$, but $\Delta E<k T$, Eq. (5) is no longer a valid approximation, and the conductivity due to the extended states of the two nearest Landau levels becomes

$$
\sigma_{x x}^{\min }=\frac{4 \sigma_{0}\left(\frac{\Delta E}{k T}\right)}{\exp \left(\frac{E_{m}-E_{f}}{k T}\right)+2},
$$

which is of a similar form to Eq. (5), except for the term $(\Delta E / k T)$ in the numerator, which acts to reduce the intercept of the conventional activation plot, producing the leveling off of $\sigma_{x x}$ observed in experiments.

We now turn to the SdeH maxima. For high $B$ and low $T$ we need only consider the contribution from the extended states of the Landau level in which the Fermi level lies. Since $E_{m}=E_{f}$, Eq. (4) reduces to

$$
\sigma_{x x}^{\max }=\frac{\sigma_{0} \sinh \left(\frac{\Delta E}{k T}\right)}{1+\cosh \left(\frac{\Delta E}{k T}\right)} .
$$

It can be seen from this expression that the $T$ dependence of the maxima is weak compared to that of the minima, and so cooling the sample makes the minima drop towards zero, increasing the size of the oscillations, while the peak resistances are relatively constant, as we observe experimentally. A natural consequence of this is the accompanying reduction in the width of the oscillation peaks as $T$ decreases.

In the final section we make some comparisons with our experimental data. ${ }^{11}$ However, before doing this it is necessary to briefly discuss the contribution of the localized states to the conductivity. We have shown that the 2D DOS consists of Landau levels with a band of extended states near the center and localized states in the tails, even at high filling factors. In such a situation we might expect there to be large overlap of the localized states, giving rise to a significant contribution to the conductivity in the minima from variable range hopping (VRH). Indeed, Polyakov and Shklovskii ${ }^{18}$ have argued that in such circumstances it should be the only mechanism for conduction. The VRH conductivity is given by Efros and Schklovskii to be ${ }^{19}$

$$
\sigma_{x x}^{h}=\sigma_{0}^{h} \exp \left(-\sqrt{\frac{T_{0}}{T}}\right),
$$

where $T_{0}$, the characteristic hopping temperature, is a measure of the density of (localized) states between adjacent Landau-level peaks. As the Landau-level overlap increases, $T_{0}$ decreases according to ${ }^{18}$ 

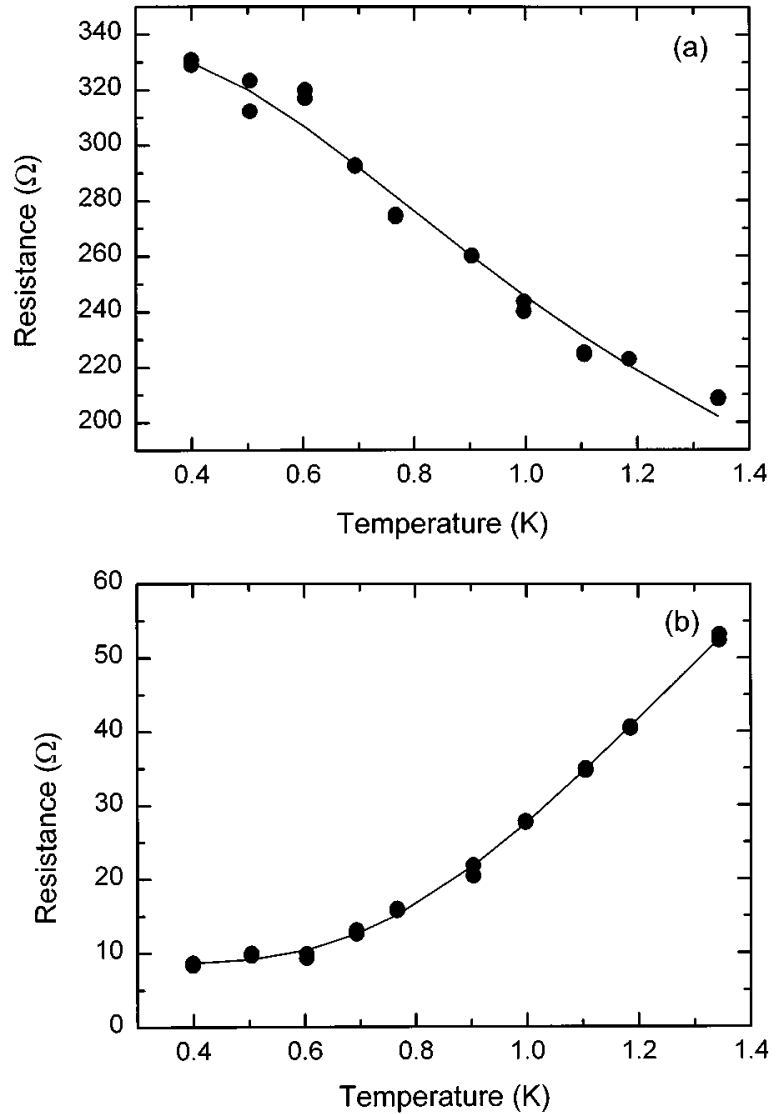

FIG. 4. $T$ dependence of the SdeH data from Ref. 11. Note that in fields where oscillations are resolved $\rho_{x x} \propto \sigma_{x x}$. (a) Maxima at $\nu=23$ ( $B=0.532 \mathrm{~T}$, points). The fit to Eq. (7) (line) gives $\Delta E$ $=1.86 \mathrm{~K}$ and a prefactor of $336 \Omega$. (b) Minima at $\nu=24$ $(B=0.51 \mathrm{~T})$. The fit to Eq. (4) gives $\Delta E=1.58 \mathrm{~K}$, a prefactor of $395 \Omega$ and a constant contribution due to VRH of $8.6 \Omega$.

$$
k T_{0} \sim \frac{e^{2}}{\varepsilon \xi}\left(\frac{E_{g}}{\Gamma}\right)^{2 \gamma},
$$

thereby increasing the VRH conductivity. Here $\varepsilon$ is the dielectric constant, $\xi$ is the localization length, $E_{g}$ is the energy gap, $\Gamma$ is the Landau-level broadening, and $\gamma \approx 2.3 .{ }^{13,18}$ If we take $E_{g} \propto B$ and $\Gamma \propto \sqrt{B},{ }^{20}$ we find that $T_{0} \sim B^{2.3}$, and so drops off very rapidly at low fields, making hopping conduction increasingly important. A field independent $\Gamma$ would enhance this trend even further. ${ }^{21}$

\section{DISCUSSION}

In this section we draw together the experimental and theoretical results presented in the preceding sections and discuss the implications for the interpretation of $\mathrm{SdeH}$ oscillations of 2DES's. We start by demonstrating that the temperature dependence of the low- $B \mathrm{SdeH}$ oscillations is consistent with the model introduced in Sec. III.

In Fig. 4(a) we show a fit of Eq. (7) to the measured $T$ dependence of the maximum at $\nu=23$ for the same sample as the data of Fig. 3. Figure 4(b) is the $T$ dependence of the adjacent minimum at $\nu=24$, with a fit to Eq. (4) with $E_{n}$ $-E_{f}$ constrained to be $\frac{1}{2} \hbar \omega_{c}$ to represent the contribution from adjacent extended states, and a constant term to account

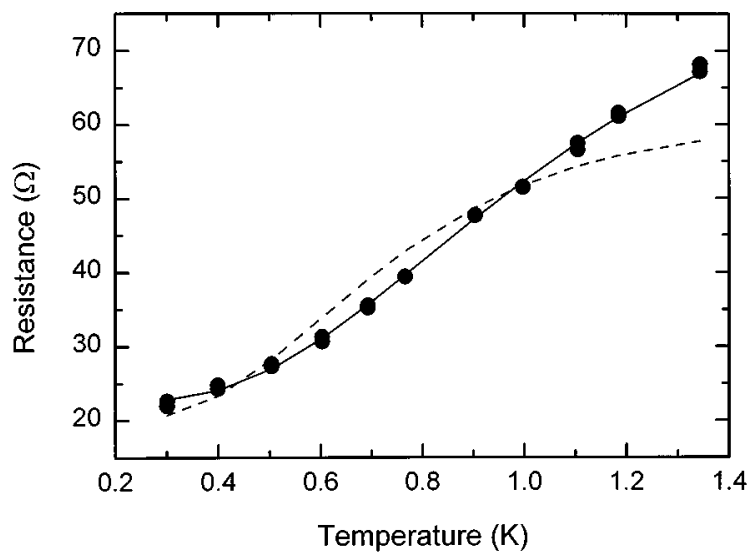

FIG. 5. $T$ dependence of the minima at $\nu=56(0.219 \mathrm{~T})$. The dashed line is an attempt to fit the data with $E_{n}-E_{f}=\frac{1}{2} \hbar \omega_{c}$, and gives $\Delta E=0.05 \mathrm{~K}$. The solid line, which is a fit with $E_{n}-E_{f}$ as a free parameter, is seen to give a much better result, with $\Delta E$ $=1.70 \mathrm{~K}$ and $E_{n}-E_{f}=3.79 \mathrm{~K}$. The contributions from VRH for the two fits are found to be 20 and $23 \Omega$, respectively.

for the contribution of VRH. The latter approximation is justified on the grounds of the narrow temperature range of the data, and the fact that extended states dominate the conductance at high $T$. In fact, attempts to fit the minimum using Eq. (8) to describe the VRH simply give $T_{0} \approx 0$. In both Figs. 4(a) and 4(b) the fits can be seen to reproduce the form of the data, strongly supporting our analysis.

We note that the two independent fits give values of $\Delta E$ of $1.86 \mathrm{~K}$ for $\nu=23$ and $1.58 \mathrm{~K}$ for $\nu=24$, a difference of about $18 \%$. We believe that most of this discrepancy is due to the levitation of extended states, ${ }^{6,7}$ which increases $E_{n}$ $-E_{f}$. As a result, if we force $E_{n}-E_{f}$ to take too small a value $\left(\frac{1}{2} \hbar \omega_{c}\right)$, then the fitting procedure will return a diminished value of $\Delta E$. For the data of Fig. 4(b) the resulting fit remains quite good, but at lower $B$ constraining $E_{n}-E_{f}$ to $\frac{1}{2} \hbar \omega_{c}$ produces implausibly low values of $\Delta E$, and an unsatisfactory fit. This is shown in Fig. 5 for data at $\nu=56$ with a dashed line giving the fit to Eq. (4) with $E_{n}-E_{f}$ constrained to be $\frac{1}{2} \hbar \omega_{c}$; it shows very poor agreement with the experimental data. In a comparable, but much more severe way as in Fig. 4(b), the fit has attempted to account for the unexpectedly large energy gap by reducing $\Delta E$ to the ridiculously low value of $0.05 \mathrm{~K}$. Moreover, this has the side effect of producing the saturation of the conductivity in the fit [see Eq. (6)], which is not seen in the data since the real broadening is much larger. A much better result is given by allowing $E_{n}$ $-E_{f}$ to vary (solid line). This gives $\Delta E=1.7 \mathrm{~K}$, which agrees very well with the high-field values of Fig. 4, and $E_{n}-E_{f}=3.79 \mathrm{~K}$, which at $0.85 \hbar \omega_{c}$ is much higher than the nominal value of $\frac{1}{2} \hbar \omega_{c}$. We have seen the same trend over a wide range of $\nu$, as summarized in Fig. 6, which presents $E_{n}-E_{f}$ in units of the cyclotron energy as a function of $B$. There are several aspects of this data which should be borne in mind. First, the energy difference obtained from these fits is to the nearest band of extended states, which could be above or below $E_{f}$. It is not possible to determine the separation between bands of extended states. Second, according to Eq. (5), it is not possible to distinguish between $E_{n}-E_{f}$ and $\Delta E$ at low $T$, leading to the possibility that both $\Delta E$ and 


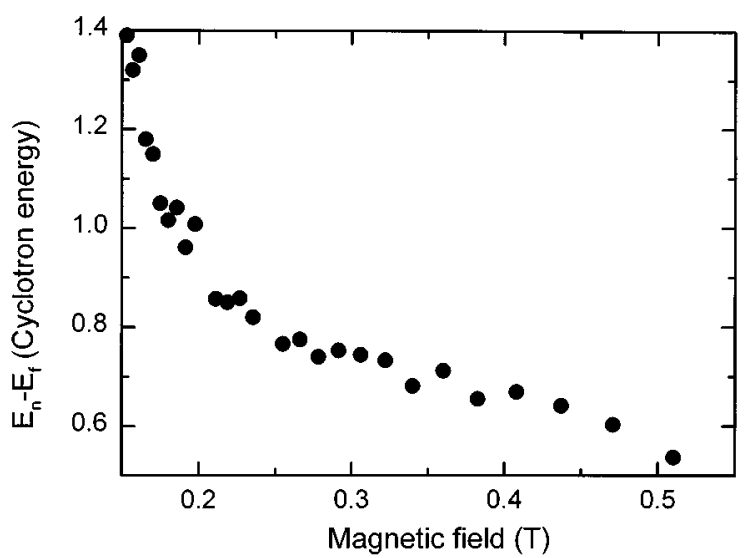

FIG. 6. Fitted values of $E_{n}-E_{f}$ for SdeH minima revealing the levitation of the extended states (solid points).

$E_{n}-E_{f}$ can be overestimated by the same amount. However, despite these drawbacks, the trend of the data is clear. $E_{n}$ $-E_{f}$ tends towards $\frac{1}{2} \hbar \omega_{c}$ at high $B$, and diverges as $B$ goes to zero. We also note that because we have been able to identify the movement of the extended states, we can be confident that our results are not due to the downward shift of the mean Landau-level energy predicted by Haldane and Yang. ${ }^{7}$ Indeed, since our results are obtained in the limit of large $n$ and low $B$, their theory would imply that the levitation effect is dominant anyway. Clearly, if the extended states are levitated, they do not necessarily correspond with the maxima in the DOS, and the peak in the resistance may no longer correspond to the center of a region of extended states. This may also be discerned in our data using our analysis, however, the very weak nature of the $T$ dependence of the maxima at low $B$ makes this method of detecting the movement less reliable than for the minima.

We are now in a good position to understand the interplay between the quantum and the semiclassical descriptions of the SdeH oscillations of 2DES's. At high $B$, that is to say, when the extended states are at, or very close to, the peaks in well separated Landau levels then only the quantum picture we have discussed can be used to describe the oscillations. At lower $B$, the Landau levels begin to overlap, increasing the contribution played by the localized states to the point where the DOS begins to look like one of the equivalent states used in the semiclassical description, and the data forms a Dingle plot (Fig. 3). However, even in this regime, examining the $T$ dependence of the data tends to bring out the contribution of the extended states, simply because it is much stronger than for the localized states. The fact that the semiclassical approach fails most spectacularly in samples with high mobility and low density, i.e., those in which the fractional quantum Hall effect is most easily observed, ${ }^{12}$ is now readily understood. For low mobility samples in which $\Delta E$ is very narrow, and which there is a large overlap of localized states, one would indeed expect the semiclassical picture to remain valid to much higher $B$.

Finally, we should like to comment on the use of Eq. (1) to determine the effective masses of composite fermions by analysis of the SdeH oscillations around $\nu=1 / 2$. Several difficulties with this approach have already been pointed out. ${ }^{4}$ Given the discussion presented above, and bearing in mind that such measurements are conducted in low-density, highmobility samples for which the same approach fails most spectacularly for the electrons, ${ }^{12}$ the implications of these types of experiments must be carefully considered. Indeed, the mere fact that both activation and semiclassical [Eq. (1)] approaches have been used to interpret data from the same samples $^{2,4}$ raises some interesting questions about the issue.

\section{CONCLUSIONS}

We have observed steplike changes in the Hall resistance of the 2DES in a GaAs/ $\mathrm{Al}_{x} \mathrm{Ga}_{1-x} \mathrm{As}$ heterojunction when continuously illuminated with RLED and IRLED in a static magnetic field, with plateaus at even integer filling factors from 22 to 80 . We attribute this behavior to the quantum Hall effect, and conclude that even at the high filling factors normally considered appropriate for a semiclassical analysis of the amplitude of SdeH oscillations, the DOS in a magnetic field consists of Landau levels with a band of extended states near the center, and localized states in the tails. This explains the failure of the semiclassical approach to describe the $T$ dependence of SdeH oscillations in similar samples. From general considerations of the contribution to the conductivity from both extended and localized states we have developed an alternative description of the $T$ dependence of the maxima and minima of SdeH oscillations appropriate in both high and low $B$. We have shown our approach to be in good qualitative agreement with experimental observations, and used it to reveal the predicted levitation of extended states.

\section{ACKNOWLEDGMENTS}

This work was supported by the Engineering and Physical Sciences Research Council, U.K. M.H. is currently supported by the F.W.O. (Belgium).
${ }^{1}$ T. Ando, J. Phys. Soc. Jpn. 37, 1233 (1974); A. Isihara and L. Smrčka, J. Phys. C 19, 6777 (1986); P. T. Coleridge, R. Stoner, and R. Fletcher, Phys. Rev. B 39, 1120 (1989).

${ }^{2}$ R. R. Du, H. L. Stormer, D. C. Tsui, L. N. Pfeiffer, and K. W. West, Phys. Rev. Lett. 70, 2944 (1993); Solid State Commun. 90, 71 (1994).

${ }^{3}$ D. R. Leadley, R. J. Nicholas, C. T. Foxon, and J. J. Harris, Phys. Rev. Lett. 72, 1906 (1994); D. R. Leadley, M. van der Burgt, R. J. Nicholas, C. T. Foxon, and J. J. Harris, Phys. Rev. B 53, 2057
(1996); R. R. Du, H. L. Stormer, D. C. Tsui, L. N. Pfeiffer, and K. W. West, Phys. Rev. Lett. 73, 3274 (1994); H. C. Manoharan, M. Shayegan, and S. J. Klepper, ibid. 73, 3270 (1994); P. J. Gee, F. M. Peeters, J. Singleton, S. Uji, H. Aoki, C. T. Foxon, and J. J. Harris, Phys. Rev. B 54, R14 313 (1996).

${ }^{4}$ P. T. Coleridge, Z. W. Wasilewski, P. Zawadski, A. S. Sachrajda, and H. A. Carmona, Phys. Rev. B 52, R11 603 (1995).

${ }^{5}$ For review, see, R. E. Prange and S. M. Girvin, The Quantum Hall Effect, 2nd ed. (Springer-Verlag, New York, 1990). 
${ }^{6}$ R. B. Laughlin, Phys. Rev. Lett. 52, 2304 (1984); D. E. Khmelnitskii, Phys. Lett. 106A, 182 (1984); T. V. Shahbazyan and M. E. Raikh, Phys. Rev. Lett. 75, 304 (1995).

${ }^{7}$ F. D. M. Haldane and K. Yang, Phys. Rev. Lett. 78, 298 (1997).

${ }^{8}$ I. Glozman, C. E. Johnson, and H. W. Jiang, Phys. Rev. Lett. 74, 594 (1995); S. V. Kravchenko, W. Mason, J. E. Furneaux, and V. M. Pudalov, ibid. 75, 910 (1995).

${ }^{9}$ M. Hayne, A. Usher, J. J. Harris, and C. T. Foxon, Surf. Sci. 362, 574 (1996).

${ }^{10}$ P. M. Mooney, Semicond. Sci. Technol. 6, B1 (1991).

${ }^{11}$ M. Hayne, A. Usher, J. J. Harris, and C. T. Foxon, Phys. Rev. B 46, 9515 (1992).

${ }^{12}$ P. T. Coleridge, M. Hayne, P. Zawadzki, and A. Sachrajda, Surf. Sci. 362, 560 (1996).

${ }^{13}$ S. Koch, R. J. Haug, K. von Klitzing, and K. Ploog, Phys. Rev. B 46, 1596 (1992).
${ }^{14}$ N. R. Cooper and J. T. Chalker, Phys. Rev. B 48, 4350 (1993).

${ }^{15}$ D. G. Polyakov and M. E. Raikh, Phys. Rev. Lett. 75, 1368 (1995).

${ }^{16}$ L. Smrčka and P. Středa, J. Phys. C 10, 2153 (1977).

${ }^{17}$ A. Usher, R. J. Nicholas, J. J. Harris, and C. T. Foxon, Phys. Rev. B 41, 1129 (1990).

${ }^{18}$ D. G. Polyakov and B. I. Shklovskii, Phys. Rev. B 48, 11167 (1993).

${ }^{19}$ A. L. Efros and B. I. Shklovskii, J. Phys. C 8, L49 (1975).

${ }^{20}$ T. Ando and Y. Uemura, J. Phys. Soc. Jpn. 36, 959 (1974).

${ }^{21}$ M. E. Raikh and T. V. Shahbazyan, Phys. Rev. B 47, 1522 (1993); A. Potts, R. Shepherd, W. G. Herrenden-Harker, M. Elliot, C. L. Jones, A. Usher, G. A. C. Jones, D. A. Ritchie, E. H. Linfield, and M. Grimshaw, J. Phys. C 8, 5189 (1996). 